Originalveröffentlichung in: Études germaniques, 64 (2009), Nr. 4. S. 1037-1053

Études Germaniques 64 (2009), 4, p. 1037-1053

Mathilde ARNOUX*

\title{
Art Allemagne Aujourd'hui ou la reconnaissance de l'art allemand contemporain par les musées français
}

In 1981 the musée d'Art moderne de la Ville de Paris organized the exhibition Art Allemagne Aujourd'hui which presented for the first time in France a large display of German contemporary art. This event was set up as a reconciliation with contemporary German culture, which was long rejected because of the hatred of some French people, but also of the traces of the World War II. Through the analysis of the catalogs' text, of the choices of works and artists, we attempted to understand what was shown to the French. Which german artists were chosen to represent contemporary Germany in the context of the Cold War, which symbols did the exhibition finally attempt to embody?

1981 veranstaltete das « Museum für moderne Kunst der Stadt Paris » eine Ausstellung (Art Allemagne Aujourd'hui), die sich zum erstenmal zum Ziel setzte, ein Panorama der zeitgenössischen Kunst in Deutschland vorzustellen. Erstrebt wurde auf französischer Seite eine Versöhnung mit der zeitgenössischen deutschen Kultur, die in Folge der langjährigen Feindschaft, aber auch der vom Zweiten Weltkrieg hinterlassenen Spuren in Deutschland selbst missverstanden und verworfen wurde. Im vorliegenden Aufsatz wird von der Analyse der Katalogtexte, der Wahl der ausgestellten Werke und Künstler ausgegangen. Damit wird versucht, das, was dem französischen Publikum präsentiert wurde, genauer zu verstehen. Welche deutschen Maler sollten im damaligen Kontext des Kalten Krieges das zeitgenössische Deutschland repräsentieren? Welche Symbole wurden durch die Kuratoren der Ausstellung in den Vordergrund gerückt? Hervorgekehrt wird die Bedeutung der Rolle, die den Museen in Hinsicht auf Verbreitung und Verständnis der deutschen Kultur in Frankreich zugewiesen wurde. Gleichzeitig wird der Frage nachgegangen, ob die Wahl der Bilder und Künstler durch die französischen Institutionen dazu beitrug, die deutsche Kunst besser zu verstehen.

L'exposition Penck en 2008 aboutissait à une vitrine constituée de documents et d'archives visant à resituer la réception de l'artiste en France. Le premier document était le catalogue de l'exposition Art Allemagne Aujourd'hui, organisée en 1981, à l'ARC et au musée d'Art moderne de la ville de Paris. Cet événement apparaissait comme le point de départ de la reconnaissance de Penck en France. Bien plus encore, cette exposition est la première à avoir été entièrement con-

\footnotetext{
* Mathilde ARNOUX, docteur en histoire de l'art, est Directrice de Recherche au Centre allemand d'histoire de l'art, 10 place des Victoires, F-75002 PARIS; courriel : marnoux@dtforum.org
} 
sacrée à l'art allemand de la seconde moitié du XX $\mathrm{XX}^{\mathrm{e}}$ siècle dans un musée français et à avoir présenté au public une sélection d'artistes aujourd'hui encore considérés comme les jalons de l'histoire de l'art allemand des années 1960 et 1970. Parmi eux se trouvaient les figures alors incontournables de Josef Beuys et Wolf Vostell, ainsi que Sigmar Polke, Gerhard Richter, Hanne Darboven, Palermo ou encore Klaus Rinke, c'est par ailleurs une des premières expositions d'art allemand à l'étranger qui affichait l'importance de la jeune peinture à travers la présentation d'œuvres de Georg Baselitz, A.R. Penck, Markus Lüpertz ou encore Jörg Immendorff. ${ }^{1}$

Certains artistes allemands contemporains avaient déjà été présentés en France à travers des expositions monographiques ou de groupes dans divers musées, Saint-Étienne, l'ARC, le Musée national d'art moderne, ${ }^{2}$ la galerie Bama et la Galerie Gillespie-Laage-Salomon à Paris avaient développé une prédilection pour la peinture allemande contemporaine; mais personne ne s'était encore risqué à proposer une synthèse de l'art allemand contemporain. Cette période était de loin l'une des plus difficiles à aborder puisqu'elle s'attachait à l'Allemagne d'après-guerre, à une culture postérieure au nazisme et par rapport auquel elle devait nécessairement se situer étant donné les marques qu'il avait laissées. Les musées français ne consacraient des expositions à l'art allemand que depuis les années 1950. Elles sont mises au service des relations culturelles franco-allemandes et leur portée symbolique est très forte. Chacune doit incarner une nouvelle étape dans la connaissance et la compréhension de la culture allemande. ${ }^{3}$ Ainsi, après l'exposition sur le romantisme allemand qui avait fait découvrir la peinture d'une période déjà admirée par Mme de Staël, après Paris-Berlin qui avait ouvert les yeux du public sur un aspect de la culture allemande longtemps craint et ignoré, Art Allemagne Aujourd'hui se penchait sur l'Allemagne contemporaine.

L'exposition s'inscrit dans une remarquable programmation culturelle organisée avec l'Institut Goethe de janvier à mars 1981. Celle-ci vise à donner un aperçu de la production artistique allemande contemporaine. Parallèlement à l'exposition organisée par Suzanne Pagé,

1. Cet intérêt pour la jeune peinture allemande est manifeste dans diverses expositions organisées la même année en Europe. Voir à ce sujet Schilderkunst in Duitsland 1981. Peinture en Allemagne, cat. exp. Bruxelles, Société des expositions du Palais des BeauxArts de Bruxelles, 1981; A New Spirit in Painting, cat. exp. Londres, Royal Academy, 1981.

2. Vostell. Environnements/Happenings 1958-1974, cat. exp., Paris, musée d'Art moderne de la ville de Paris, 1974; Impressions artistiques de la R.D.A. d'aujourd'hui. Peintures et sculptures, cat. exp. Lille, Palais des Beaux-Arts, 1975; Gerhard Richter, cat. exp., Paris, Centre national d'art et de culture Georges-Pompidou, 1977; Après le classicisme, cat. exp. Saint-Étienne, 1980.

3. Mathilde Arnoux : Les musées français et la peinture allemande 1871-1981, Paris: Éditions de la MSH, 2007, chap. VII et IX. 
conservateur du musée d'art moderne de la ville de Paris, directrice de l'ARC en collaboration avec le galeriste et commissaire d'exposition René Block, l'Institut Goethe propose une programmation dense de projections cinématographiques, de conférences sur la littérature contemporaine et de lectures par les auteurs, de concerts de musiques contemporaines et jazz, de théâtre. Les objectifs sont clairs, il s'agit d'offrir la possibilité de découvrir la création allemande contemporaine, de dépasser les stéréotypes appliqués au voisin d'outre-Rhin, inquiétant, incompris, rejeté en raison de la haine ou de l'ignorance de certains Français mais aussi de la violence des marques de la Seconde Guerre mondiale qui imprègne l'Allemagne d'après-guerre. ${ }^{4}$

Ces manifestations culturelles ont une fonction diplomatique forte. Elles consacrent le pas fait en direction de l'ennemi d'autrefois, elles marquent le dépassement de certaines haines et craintes, elles incarnent aussi la reconnaissance d'une possible culture allemande sortie des années de nazisme, elles témoignent en France d'une recherche, d'une curiosité pour de nouveaux modèles. Elles laissent croire à une rencontre avec le voisin. Le message de réconciliation, la volonté de dépassement des stéréotypes, le désir de passer outre les rancœurs qui ont infiltré les relations franco-allemandes sont affichées, revendiquées. En revanche, il est moins évident d'apprécier les contours de ce voisin à la rencontre duquel on se dirige la main tendue. Que portait en elle cette présentation de l'Allemagne contemporaine?

Afin de comprendre ce que cette exposition a pu transmettre de la culture allemande, il est essentiel de s'interroger sur ce qui allait incarner l'Allemagne qui s'affichait fièrement au titre de l'exposition de l'ARC. Quelle part le contexte politique a-t-il joué dans la définition et la compréhension de l'art allemand contemporain? Plus précisément encore, les enjeux idéologiques issus de la Guerre froide ont-ils eu un impact sur la présentation de l'Allemagne contemporaine? Ces questions doivent être soulevées pour tenter de comprendre les éléments à partir desquels se fonde l'image de l'autre et de saisir l'étroite imbrication qu'il peut y avoir entre les domaines culturels et politiques, d'apprécier combien notre connaissance en est tributaire, dépendante. Cette conscience aiguë de ce qui est transmis ou au contraire éludé de la culture du voisin doit être impérativement interrogé aujourd'hui. Ces choix ont en effet été fortement déterminés par la Guerre froide et instrumentalisés par chacun des modèles, le capitaliste comme le socialiste. Nous sommes aujourd'hui héritiers des effets de cette langue de bois et si les anciens pays de l'Est se sont ouverts de gré ou de force à l'Ouest, l'Ouest ignore encore largement la diversité de ce qui se pro-

4. Suzanne Pagé, texte introductif dans: Art Allemagne Aujourd'hui. Différents aspects de l'art actuel en République Fédérale d'Allemagne, cat. exp. Paris, ARC/Musée d'art moderne de la ville de Paris, 1981, p. 5. 
duisait à l'Est, comme en atteste d'ailleurs l'absence quasi totale de la présentation de l'art produit à l'Est du rideau de fer dans la plupart des manuels et synthèses d'histoire de l'art (mis à part peut-être pour ce qui est du réalisme socialiste). Les questions soulevées par l'analyse de l'exposition Art Allemagne Aujourd'hui doivent conduire à une réflexion plus approfondie sur ce qui s'est joué à l'Ouest face ou en réaction aux ambitions artistiques présentées par l'Est.

\section{Quelle Allemagne l'exposition donne-t-elle à voir?}

Art Allemagne Aujourd'hui est organisée par Suzanne Pagé, alors conservateur et directrice de l'ARC. Pour définir sa sélection d'œuvres, le choix des artistes, elle s'est fait conseiller par ses amis artistes dont Wolff Vostell auquel elle avait déjà consacré une exposition en 1974, des professeurs des écoles des Beaux-Arts Richter, Beuys lui-même, mais surtout et essentiellement par René Block. ${ }^{5}$ Celui-ci avait ouvert une galerie en 1964 à Berlin pour y présenter l'art contemporain, notamment Gerhard Richter, Sigmar Polke, Josef Beuys, Wolf Vostell, Nam June Paik. À partir des années 1970, il devient un important commissaire indépendant. En 1974, il inaugure un espace d'exposition à New York en y présentant la fameuse performance de Beuys I like America and America likes me. René Block est alors un acteur essentiel de la scène artistique contemporaine. L'exposition, qui aurait dû être inaugurée en novembre 1980, est reportée à plusieurs reprises pour pouvoir tenir lieu de décor à la rencontre entre Valéry Giscard d'Estaing et Helmut Schmidt dans le cadre du sommet franco-allemand organisé en février 1981 et essentiellement consacré aux relations culturelles entre les deux pays. La toile de fond est donc d'emblée hautement politique et diplomatique. Ce caractère politique et idéologique est d'ailleurs analysé très adroitement par l'un des artistes, Hans Haacke, dans un petit texte publié dans le catalogue et à l'appui duquel il explique ce qui a déterminé le choix de l'œuvre qu'il présente. ${ }^{6}$

Afin de comprendre plus précisément les ambitions de cette exposition, il ne reste plus aujourd'hui que quelques pièces d'archives et surtout le catalogue de l'exposition. ' L'introduction de Suzanne Pagé est claire et affiche d'entrée de jeu les intentions de la manifestation. Il s'agit de présenter l'Allemagne allemande et, à l'exception de Hans Haacke, sont exclus les artistes étrangers exerçant en Allemagne comme par exemple Antes, de même que les artistes allemands travaillant à l'étran-

5. Archives du Musée d'art moderne de la ville de Paris, exposition Art Allemagne Aujourd'hui, dossier 364 (1)-(3).

6. Hans Haacke dans : Art Allemagne Aujourd'hui (note 4), p. 166-167.

7. Suzanne Pagé, texte introductif dans : Art Allemagne Aujourd'hui (note 4), p. 5. 
ger tels que Jochen Gerz, Klasen... L'objectif est de mettre en valeur la singularité allemande ignorée des Français chez lesquels l'Allemagne suscite encore des réactions passionnelles. Le ton est ferme, il s'agit d'éclairer les raisons du rejet et de l'ignorance de cette scène allemande en France, en repérant les stéréotypes appliqués à l'art allemand et en les combattant à travers des analyses nuancées distinguant les diverses pratiques avec finesse.

Mis à part le « cas Beuys », isolé comme un phénomène hors temps selon la volonté même de l'artiste qui a piloté à l'échelle internationale la réception de sa carrière ${ }^{8}$ Suzanne Pagé distingue la génération d'avant 1968, marquée par l'engagement, et celle d'après 1968, caractérisée par la désillusion. Elle rappelle que si l'art de la première génération peut être éclairé par les grands repères internationaux, toujours de façon abusive et réductrice, on constate depuis une dizaine d'années, une autonomie de plus en plus grande par rapport aux zones d'influences internationales et notamment américaine, accompagnée par l'affirmation d'une identité propre. La diversité des pratiques, illustrées par la variété des supports présentés (peinture, sculpture, environnements, objets vidéos), mais aussi par des manières d'exercer distinctes, plaident en faveur d'une Allemagne complexe, multiple et refusent l'idée d'une prétendue germanité. Pour mieux la combattre et en souligner l'aberration du point de vue du concept, Suzanne Pagé propose d'analyser la complexité et la diversité des fondements de cette prétendue germanité. Ainsi le texte d'Antje von Graevenitz dans le catalogue se penche sur l'étude des raisons pour lesquelles certains artistes s'exportent si bien en tant qu'Allemands à l'étranger comme Wagner et Beuys, et se demande en quoi ceux-ci remplissent les attentes de l'étranger à l'égard de l'Allemagne. Suzanne Pagé, de son côté, s'interroge sur les raisons pour lesquelles la «jeune peinture » allemande et ses références culturelles ont fait l'objet de tant de rejet et de haine dans son pays d'origine alors qu'elle a été si bien accueillie par l'étranger. René Block explique quant à lui la façon dont les Allemands alimentent eux-mêmes les stéréotypes. ${ }^{9}$ Rien de cohérent ne ressort des différentes utilisations de la notion de germanité présentées dans ces textes, ce qui en invalide ainsi l'emploi avec ses sous-entendus négatifs tels que l'on en faisait usage en France.

Émancipée des terminologies réductrices, la recherche de valorisation des spécificités allemandes se fonde ainsi sur la caractérisation de l'originalité des pratiques artistiques contemporaines. Dans son introduction, Suzanne Pagé s'efforce de les distinguer sans les opposer. Si

8. Voir à ce sujet Maïté Vissault : La Problématique de l'identité allemande à travers la réception de l'œuvre de Joseph Beuys, Université de Rennes II, 2001. À paraître sous le titre : Der Beuys-Komplex. L'Identité allemande à travers la réception de Joseph Beuys (1945-1986), Dijon : Les Presses du réel, 2010.

9. René Block : "Entre Paris et Berlin : RFA », dans : Art Allemagne Aujourd'hui (note 4), p. 79. 
elle caractérise différemment la génération d'avant 1968 de celle qui suit, elle ne les présente pas à travers une confrontation radicale. Cet aspect, sur lequel nous reviendrons, est essentiel car il se distingue de l'écriture moderniste de l'histoire de l'art décrivant successivement et en opposition les unes aux autres les recherches artistiques. Par ailleurs, ce souci de présenter la variété de la scène artistique allemande contribue à la définir comme une entité unie dans sa diversité.

Cette scène artistique caractérisée par sa créativité, sa diversité, est analysée comme largement tributaire du contexte spécifique dans lequel est née la RFA. Le système politique allemand a offert une autonomie aux régions et à leurs écoles d'art garantissant la création et la variété des productions. À travers ce texte resplendissent les valeurs fondamentales attachées à la RFA qui apparaît comme un pays fondé sur un système de libre entreprise et de concurrence, moderne, créatif, autonome. Cette image est extrêmement diplomatique. Elle se situe en stricte opposition aux critiques dont la RFA avait fait l'objet en France à la fin des années 1970 et prend le contre-pied des accusations qui en faisait un État policier en raison de la vigueur de sa répression antiterroriste. ${ }^{10}$ Cet éloge du système fédéral et des valeurs qui y sont liées illustre la position officielle de la France à l'égard de la RFA et souligne de manière évidente la façon dont la culture peut être mise au service de la valorisation d'un système.

Ces enjeux diplomatiques, traditionnellement propres au domaine culturel, ne doivent cependant pas laisser croire à la soumission totale du discours du catalogue aux ambitions politiques immédiates et à une absence de nuances. Pour preuve : les différences de pratiques des artistes présentés dans le cadre de l'exposition, la variété des propos des textes du catalogue, ne permettent pas et n'ont d'ailleurs pas pour intention de donner une image strictement circonscrite de l'Allemagne présentée au travers de l'exposition, celle-ci ayant pour intention de valoriser la diversité de cette scène artistique. Le fleuve de l'art allemand dessiné par René Block et publié au catalogue est l'illustration même des nombreuses sources auxquels l'art allemand contemporain s'abreuve (ill. p. 1043).

Celui-ci est issu des avant-gardes du début du siècle : expressionnisme, Dada, Bauhaus. La Nouvelle Objectivité est en revanche exclue. Après s'être écoulé souterrainement pendant le nazisme, le fleuve refait surface. Beuys est l'un des affluents à l'avoir le plus largement nourri et de nombreux fleuves et ruisseaux se mêlent pour l'alimenter, en passant par New York et Paris.

10. Voir Henri Ménudier : «L'Allemagne à laquelle il croit », dans F. Hartweg (dir.) : $A$ Joseph Rovan, penseur et acteur du dialogue franco-allemand. Hommages pour son soixantedixième anniversaire, Paris : Documents, revue des questions allemandes, 1989, p. 95-101. 


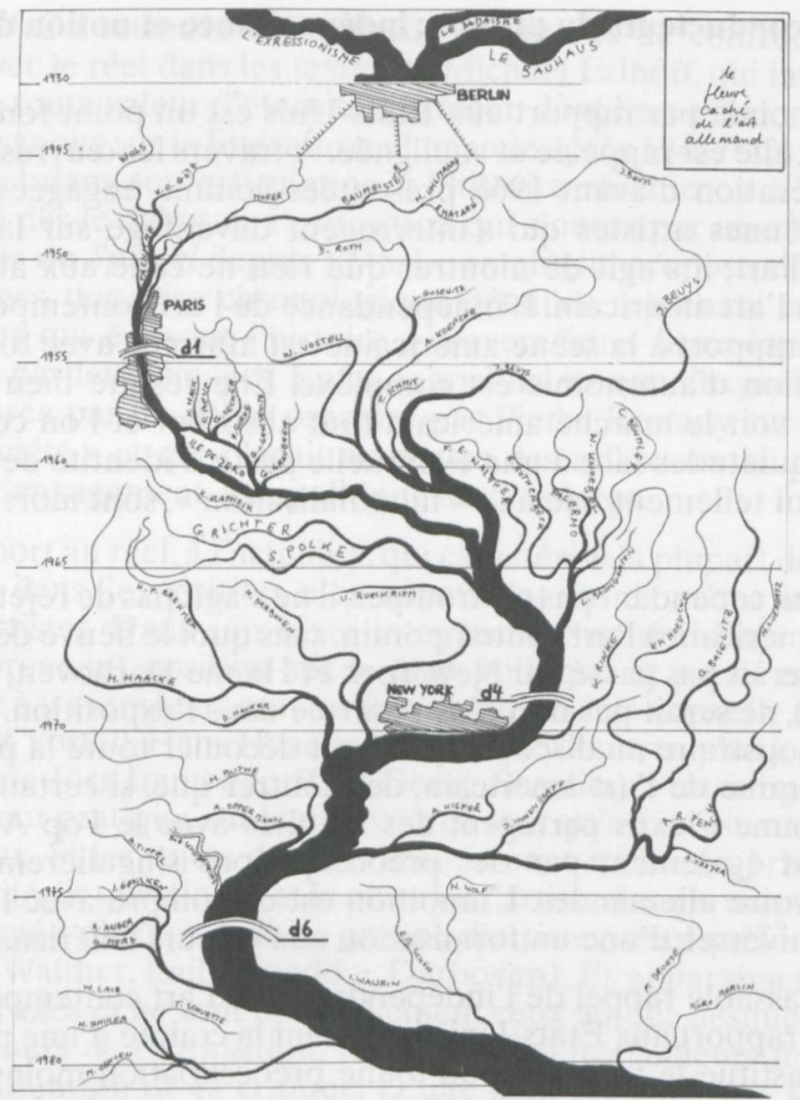

Ill. 1

Une première analyse du catalogue laisse voir que l'exposition s'attache à la présentation de l'Allemagne de l'Ouest. La modernité de sa structure fédérale est louée et analysée comme un garant de la liberté des enseignants et de l'indépendance de la création. Le caractère politique de ces considérations ne nuit néanmoins pas à l'élaboration d'un discours complexe et nuancé qui cherche à ne pas réduire les ambitions de la manifestation à la seule réconciliation franco-allemande. La lecture attentive des textes fait apparaître quelques sujets récurrents qu'il est impératif d'examiner de plus près pour mieux circonscrire ce que l'exposition montre de l'Allemagne contemporaine. 


\section{Les fils conducteurs du propos : indépendance et notion de « réel »}

L'autonomie par rapport aux États-Unis est un point fondamental du propos, elle est rappelée et soulignée. À travers les œuvres d'artistes de la génération d'avant 1968 présentées comme engagées et celles des plus jeunes artistes qui s'interrogent davantage sur la fonction même de l'art, il s'agit de montrer que rien ne cède aux attentes du marché de l'art américain. L'indépendance de l'art contemporain allemand par rapport à la scène américaine est affirmée avec force. Cette revendication d'autonomie est complexe. Elle résulte bien sûr de la crainte de voir le marché américain tout absorber et l'on constate ici que les inquiétudes face à une éventuelle perte d'identité de l'Europe, aujourd'hui tellement liée à la «mondialisation », sont alors déjà bien présentes.

Il ne faut cependant pas s'y tromper, il ne s'agit pas de rejeter en bloc l'apport américain à l'art contemporain, sans quoi le fleuve de l'art allemand ne serait pas passé par New York et Hanne Darboven, proche de Sol Lewitt, ne serait pas non plus montrée dans l'exposition. L'objectif est de se soustraire au discours qui ferait découler toute la production contemporaine de l'art américain, de montrer que, si certains mouvements comme Fluxus partagent des affinités avec le Pop Art, ils s'en distinguent également par des préoccupations singulièrement européennes, voire allemandes. L'ambition est de rompre avec l'idée d'un modèle universel, d'une uniformisation aux valeurs américaines.

Cet inlassable rappel de l'indépendance de l'art contemporain allemand par rapport aux États-Unis trahissant la crainte d'une perte identitaire, constitue la toile de fond d'une préoccupation moins affirmée, moins apparente de prime abord, et pourtant essentielle et récurrente car elle file à travers tout le catalogue et vise à saisir le lien entre les œuvres et le réel, le temps présent. Ces sujets, qui peuvent a priori paraître sans lien les uns avec les autres, trouvent pourtant une cohérence et une articulation logique.

Les œuvres, aussi variées soient-elles, sont pour la plupart mises en rapport avec la notion de réel. Celle-ci, selon les œuvres, est interprétée dans sa dimension temporelle (le réel comme actualité, comme temps présent) ou dans sa dimension représentative (le réel comme figuré par opposition à l'abstrait). Ce lien des œuvres au réel en tant qu'actualité est rappelé de manière constante. Dans l'introduction, Suzanne Pagé désigne ainsi comme caractère commun aux deux générations exposées leur « ancrage dans un contexte historique ou géographique déterminé se référant à une problématique, une réalité ou même une tradition locale, régionale ou nationale. Cet ancrage peut aussi prendre le visage de références folkloriques ou mythiques, plus ou moins obscures, qu'il conviendra d'apprécier dans cette marge étroite qui affirme que " "tout bon art est national, [que] tout art national est mauvais" (Christian 
Krogh). » ${ }^{11}$ On retrouve cette notion essentielle de confrontation de l'artiste avec le réel dans les textes de Michael Erlhoff, qui interroge ce qui enlève toute valeur d'éternité à la façon dont l'art est alors traité, et Nicole Casanova sur la littérature allemande après $1945 .{ }^{12}$ Ce lien étroit avec le réel dans son articulation à l'histoire récente ou à l'actualité est affirmé dès le début de l'exposition qui s'ouvre par un affichage de couvertures du Spiegel depuis 1945. Le catalogue propose, dès ses premières pages, une frise chronologique détaillée reprenant ces mêmes couvertures qui écrivent l'histoire à travers leurs titres lapidaires, et l'illustrent par leurs images. Enfin, rappelons ici que l'exposition avait été inaugurée par un débat organisé par Pierre Restany sur «l'artiste dans la société » et qui plaçait au centre des réflexions le rapport de l'artiste à l'engagement, à la politique au sens large.

Ce rapport au réel, à l'actualité, qui caractérise la plupart des œuvres présentées dans l'exposition, n'est cependant pas de même nature suivant les artistes. Dans son texte introductif, Suzanne Pagé distingue ceux qui prennent position sur le plan politique et social et essaient d'exprimer à travers leurs œuvres une réalité sociale nouvelle (tels Josef Beuys, Wolf Vostell, Hans Haacke), tandis que d'autres, plus jeunes souvent (comme Jörg Immendorff ou Georg Baselitz), s'interrogent sur la nature de leur pratique, sur leur devoir en tant qu'artiste, sur ce que l'on attend d'eux. Si les sujets dont ils traitent sont moins en prise directe sur l'actualité, ils questionnent la place de l'artiste dans le monde actuel. Suzanne Pagé isole un troisième groupe d'artistes, ceux qui font de l'art pour l'art (Walther, Laib, Hoedike, Darboven). Ils apparaissent cependant assez isolés et ne sont pas réellement ceux autour desquels s'organise le discours de l'exposition. Ils ne retiennent d'ailleurs finalement pas tant l'attention de la critique. D'une manière générale, le jeu sur l'ambiguïté de la notion de réel permet en fait d'articuler deux générations généralement placées en opposition l'une par rapport à l'autre : celle des années 1960 et celle qui émerge au milieu des années 1970. Un bref rappel du contexte des débats artistiques s'impose donc ici.

Face au triomphe de l'abstraction comme vocabulaire universel et symbole de la victoire de l'art moderne et de la démocratie, des artistes, dès le milieu des années 1950 , refusent les pratiques picturales traditionnelles et rejettent les ambitions de l'abstraction, aux États-Unis, en Grande-Bretagne, en France, en Allemagne, en Italie. Ils réclament un retour à la naïveté des origines, à la prise directe sur la réalité. Ils revendiquent une continuité avec les pratiques Dada mêlant collage, assemblage, performance, musique, poésie, mots, déconstruction, destruction

11. Suzanne Pagé, texte introductif dans : Art Allemagne Aujourd'hui (note 4), p. 6

12. Michael Erhoff : « Douze ans que l'on fabrique des conserves! »; Nicole Casanova, " La littérature allemande après 1945 ", dans : Art Allemagne Aujourd'hui (note 4), respectivement p. 58 et p. 62. 
et, plutôt que de porter leur intérêt sur des concepts humanistes et de s'en faire les interprètes, ils s'attachent aux objets du quotidien, aux produits de consommation et les détournent. Leurs œuvres en sont des commentaires qui se veulent plus ou moins idéologiques, politiques, qui parfois au contraire refusent catégoriquement toute connotation idéologique. Ils appartiennent au mouvement Fluxus, au mouvement Pop, aux Nouveaux Réalismes. Par les objets mêmes qu'ils utilisent (objets quotidiens, poste de télévision, détritus, voir les œuvres de Wolff Vostell), par les sujets de leurs œuvres (la consommation, les médias, les événements politiques, leur cercle intime, voir les œuvres de Gerhard Richter ou Sigmar Polke), par l'ironie, par le détournement, le réel, le présent constituent l'objet du commentaire des œuvres, c'est le sujet de leur préoccupation, de leur intérêt. À travers les événements que ces différents artistes organisent ils cherchent à dépasser le clivage entre les domaines sociopolitique et esthétique. Le présent, le réel sont également convoqués à travers la façon dont ces pratiques bouleversent les frontières traditionnelles séparant l'art de la vie. Ces pratiques, très liées à l'engagement politique des années 1960, aux utopies, à une certaine confiance dans les bienfaits du système capitaliste, vont être mises en question par une nouvelle génération d'artiste qui, eux aussi, vont se positionner par rapport au réel, mais d'une tout autre manière. Le réel entre dans leur œuvre à travers leur recours à la figuration, en rupture avec la logique des avant-gardes, ils ne recherchent pas la nouveauté absolue, leur propos, aussi complexe soit-il, est figuré (voir Anselm Kiefer, Georg Baselitz, Jörg Immendorf). La pratique de la figuration est variée. Elle utilise des figures linéaires simplifiées comme découpées dans des papiers ou à la manière de graffiti, d'autres pratiques s'inscrivent plus directement dans la suite de l'expressionnisme par les couleurs et l'utilisation de touches de peintures épaisses, d'autres renouent avec le réalisme de l'entre-deux-guerres - dit du retour à l'ordre - avec des pratiques académiques, à la recherche d'illusion. Dans ces pratiques de la figuration, le réel, dans son lien à l'actualité, n'est pas forcément l'objet du commentaire des œuvres, au contraire de ce qu'il est pour les pratiques Fluxus. Les éléments du monde réel sont mis au service de la représentation, l'œuvre s'organise à l'appui de ce qui est figuré, mais qui n'a pas forcément de lien avec l'actualité, le présent.

On a donc affaire à des pratiques qui traitent la notion de réel de manière différente et qui reposent sur l'ambiguïté même du concept, entendu comme «qui existe actuellement» (opposé à possible) ou comme "concrètement " (opposé à abstrait). Dans le cadre des pratiques artistiques présentées ici, le réel est pour certains assimilé au présent, à l'actualité, il constitue l'objet commenté, interprété, sujet des œuvres et, pour d'autres, le réel est assimilé à la figuration à laquelle on emprunte des formes pour constituer un discours mais qui n'est pas forcément lié au présent. On a donc affaire ici à des compréhensions différentes du réel, selon les artistes, et toute la période dont traite l'ex- 


\section{position Art Allemagne Aujourd'hui est très nettement aux prises avec un questionnement autour des notions de réel.}

Ce n'est certainement pas un hasard si, dès le milieu des années 1970, et au début des années 1980, alors que coexistent les pratiques néo-dada et les pratiques figuratives, on redécouvre les réalismes de l'entre-deux-guerres à travers des colloques et des expositions qui souvent tissent des liens directs entre les années 1920-1930 et la contemporanéité. ${ }^{13}$ Le rapport établi entre les deux périodes est largement contextuel (crise, violence, tension succédant à l'espoir et l'utopie), le plus souvent les textes proposent de rapprocher les pratiques figuratives de l'entre-deux-guerres de celles émergeant à partir de la seconde moitié des années $1970 .{ }^{14}$ Plus rarement, d'autres perçoivent qu'il n'y a pas opposition radicale entre figuration et démarches néo-dada ou pop et montrent que certains artistes, en dépit de pratiques différentes, répondent à des ambitions, des inquiétudes communes. Ainsi dans son compte rendu pour Art Press de l'exposition des Réalismes organisée à Beaubourg en 1980, Catherine Millet présente l'avant-garde et les pratiques Dada comme le revers du retour au réalisme et comme des réponses complémentaires, et non antithétiques, aux bouleversements

13. Voir par exemple les catalogues des expositions Les Réalismes en Allemagne, 19191933, cat. exp., musée de Saint-Étienne, 1974, Les Réalismes 1919-1939, cat. exp., Paris, Centre Georges-Pompidou, 1980, ainsi que les actes du colloque qui ont été publiés sous le titre : Le Retour à l'ordre dans les arts plastiques et l'architecture, 1919-1925, Jean-Paul Bouillon, Bernard Ceysson et Françoise Will-Levaillant (dir.), Saint Étienne : Centre interdisciplinaire d'études et de recherche sur l'expression contemporaine, 1975.

14. Voir le texte de Jean Clair : «Situation des Réalismes. De la Neue Sachlichkeit à l'Hyperréalisme ", dans : Chronique de l'Art vivant, 48 (avril 1974), p. 20-25, dans lequel l'auteur commente l'exposition organisée la même année sur les Réalismes en Allemagne, 1919-1933 au musée de Saint-Étienne, p. 24. Après un rappel de la résurgence du réalisme en Europe et aux États-Unis dans les années 1920, Jean Clair écrit dans son article : «On ne manquera pas, par conséquent, de faire la comparaison avec ce qui se passe aujourd'hui et d'être frappé par l'étroite similitude des situations. Sur le plan artistique, il semble que ce soit aujourd'hui comme autrefois, une situation marquée par l'épuisement et par la lassitude des avant-gardes comme par une sorte de lâche soulagement d'un retour au conservatisme : après les explosions d"“anti-art" et de "non-art" des années 60 , on revient à l'art, de même qu'après Dada et tous les -ismes des années 10 , on était revenu à la peinture. Sur le plan politique, parallélisme également : crise économique grave et, succédant à une agitation révolutionnaire avortée, montée un peu partout des fascismes. Ä l'apparition des fascismes espagnol, italien, allemand des années 20, correspond la naissance ou la consolidation aujourd'hui des fascismes en Amérique latine et autour du bassin méditerranéen, comme aussi bien le renforcement, autour de nous et chez nous de régimes de plus en plus musclés. C'est dans un tel contexte qu'il est nécessaire de penser la résurgence du réalisme aujourd'hui. » On retrouve des propos comparables six ans plus tard dans le catalogue de la vaste exposition intitulée Les Réalismes 1919-1939, cat. exp., Paris, Centre GeorgesPompidou, 1980, notamment dans le texte du président du Centre Georges-Pompidou, Jean Claude Groshens, qui met en relation la période contemporaine et celle des années 1920 et 1930 : « [...] Sans céder au jeu facile des parallèles, le spectateur sera sans doute plus d'une fois frappé de l'étroite similitude qu'on croit distinguer entre cette période et celle que nous vivons aujourd'hui. Peut-être même risquera-t-il de s'alarmer, à la lumière sinistre de certains événements, devant quelques-uns des documents présentés. ” 
religieux et philosophiques du $X X^{\mathrm{e}}$ siècle..$^{15}$ On ne peut s'empêcher d'y voir un reflet des questions que soulève depuis les années 1970 le retour à la figuration succédant aux pratiques néo-dada. En effet, la succession chronologique de ces pratiques artistiques, la façon dont la figuration a été comprise, surtout en Europe, en opposition à Fluxus, au Pop et aux Nouveaux Réalistes, ont conduit la plupart des historiens de l'art familiers d'une écriture progressiste et positiviste à une présentation en stricte opposition de ces mouvements. Pourtant, il apparaît aujourd'hui évident que si, d'un point de vue formel, et dans la manipulation des concepts et notamment de celui de réel, ces différents artistes n'ont pas forcément les mêmes pratiques, certains d'entre eux peuvent partager des soucis identiques, des ambitions communes. On retrouve dans beaucoup de ces œuvres la préoccupation de définir des repères identitaires, que ce soit en utilisant, en détournant, les objets de consommation produits par le monde contemporain, ou en ayant recours à des références culturelles, à des citations historiques. Ce souci s'applique aux points de

15. Catherine Millet : «Un trouble de mémoire sur le plateau Beaubourg », dans : Art Press, 43 (décembre 1980), p. 14-16. L'auteure thématise l'absence de rupture nette qu'il y a entre les avant-gardes et le retour au réalisme de l'entre-deux-guerre, de même qu'il n'y a pas de scission nette entre une tradition que l'on ferait découler de Dada et l'autre de la Nouvelle Objectivité : p. 14 « [...] opposer, comme le fait l'actuelle exposition aux avant-gardes du recommencement à 0 , les courants réalistes qui, dans les années 20 et 30 , cherchèrent à renouer avec la fonction représentative de la peinture, à recouvrer un métier traditionnel et le sens de l'héritage, c'est délibérément gommer la complexité des avantgardes, c'est avoir de celles-ci une vision aussi réductrice et abusive que leurs manifestations extrémistes eurent de la tradition. C'est reconduire un clivage qui n'est qu'une vue de l'esprit, - autant dire une stratégie.

Au sein de toutes les écoles modernes, chez tous les artistes, on trouve les traces de ce balancement, de cette dialectique entre le désir d'un art originel et l'ardeur à exhumer un passé que l'académisme du XIX ${ }^{\mathrm{e}}$ avait dissimulé ou perverti. Le premier mouvement ne va pas sans le second. ». Après avoir tracé en quelques phrases le contexte de la création dans l'entre-deux-guerres, Catherine Millet explique p. 15: «Dada n'est que le signe en négatif d'une stabilisation et d'une normalisation que les réalismes expriment en positif. Deux pôles qui marquent, nous allons le voir, une même limite. [...] La crise des valeurs spirituelles et la domination de plus en plus étendue de la pensée marxiste sont les deux bornes sur lesquelles bien des œuvres au XX $\mathrm{XX}^{\mathrm{e}}$ siècle sont venues buter. Il n'y a finalement pas tant de différence entre un dadaïste qui, ayant cessé de croire en la spécificité de l'art, dissout celui-ci dans un activisme militant, et un peintre réaliste qui, lui aussi limitant son art à une fonction sociale, le met au service d'un discours. Il n'y en a pas non plus entre tous ceux qui, cherchant à éviter cette soumission, ne surent qu'opérer des retours en arrière, qu'il s'agisse de Marcel Duchamp qui efface le tableau comme mémoire du monde et qui, devenu prisonnier de l'objet, ne sait y échapper que dans l'ésotérisme, ou qu'il s'agisse de De Chirico réagissant au pragmatisme futuriste en engageant le dialogue avec les fantômes. Jean Clair qui fait de cette partie de l'œuvre de Duchamp et de celle de De Chirico les pôles de sa démarche critique. confirme à quel point l'un et l'autre sont deux points symétriques sur un même vecteur. Les écoles réalistes et le mouvement de « retour aux sources» ne sont pas la contradiction de l'avant-gardisme, ils en sont au contraire étroitement solidaires dans un même ressassement des limites de l'art au XXe siècle [...] Avant-gardismes et réalismes sont venus colmater les profonds bouleversements religieux et philosophiques qui inaugurèrent le siècle, empêcher qu'ils soient pensés dans leur dimension véritable. » Voir également au sujet de la frontière conventionnellement établie entre pratique d'avant-garde et retour au classicisme le texte de Jill Carrick : "Le Nouveau Réalisme : un détournement de la profusion des choses », dans : Nouveau Réalisme, cat. exp., Paris, Galeries nationales du Grand Palais, 2007, p. 176-181. 
repères sur lesquels peut se fonder l'identité après les bouleversements éthiques et philosophiques engendrés par le second conflit mondial.

L'exposition organisée par Suzanne Pagé est de ce point de vue exemplaire, dans sa capacité à rassembler ces pratiques diverses et variées et dans sa recherche à les saisir comme un ensemble. Mais il est un point qu'il faut cependant chercher à éclairer : les raisons pour lesquelles, alors qu'elle accepte aussi bien les pratiques Fluxus que la jeune génération de peintres ayant recours à la figuration, elle refuse ce qui pourtant émerge depuis le début des années 1970 : une pratique liée dans ces recherches au réalisme de l'entre-deux-guerres. Pourquoi ne les intègre-t-elle pas, alors que la galerie Poll lui propose des œuvres de Klaus Vogelgesang ou Wolfgang Petrick qui réagissent contre le Pop et les valeurs de la bourgeoisie et mettent l'hyperréalisme au service d'une description crue de la société.

\section{Des choix conditionnés par la Guerre froide}

Si certains partis pris semblent assez clairement expliqués dans le texte introductif, comme l'absence des Allemands vivant à l'étranger ou des étrangers vivants en Allemagne, on s'étonnera davantage de l'absence des tendances permettant d'établir un lien entre la peinture contemporaine allemande et la Nouvelle Objectivité. On retrouve ici la question déjà soulevée par le fleuve caché de l'art allemand qui ne faisait pas figurer la Nouvelle Objectivité parmi les fondements de l'art allemand contemporain.

Cette absence doit être interprétée comme le reflet des questions soulevées par le problème des identités artistiques respectives de l'Est et de l'Ouest. Si les deux types de pratique (figuration et pratique néodada) coexistent en réalité dans chacun des blocs, l'Est n'est officiellement autorisé qu'à présenter des tendances relevant du réalisme dit socialiste, le seul à être reconnu par le régime. L'Ouest présente la diversité des pratiques qu'il autorise comme une incarnation des libertés d'expression, d'engagement, de subversion, la reconnaissance de l'individu. L'affirmation si forte du réel comme objet de préoccupation des artistes allemands contemporains, s'oppose en fait au réalisme tel qu'il est pratiqué à l'Est. Cette distinction radicale entre deux pratiques artistiques opposant l'Est à l'Ouest est factice. Cependant, comme nous l'avons déjà compris, Art Allemagne Aujourd'hui valorise la RFA. Elle est organisée peu de temps avant une exposition hautement diplomatique sur l'art contemporain de $\mathrm{RDA}^{16}$ et il était donc fondamental de bien distinguer chaque Allemagne dans sa pratique artistique.

16. Le texte introductif de Bernadette Contensou, conservateur au Musée d'Art moderne de la ville de Paris, semble avoir été dicté par le Ministère des Affaires Étrangères 
Or, les réminiscences de la Nouvelle Objectivité sont nettement associées à la RDA. Le terme de « réalisme » est d'emblée utilisée par Hans Joachim Hoffman, ministre de la culture de RDA dans son avantpropos, ainsi que par les autres auteurs du catalogue. ${ }^{17}$ En excluant toutes les pratiques trahissant des préoccupations communes à l'Ouest et à l'Est, et notamment ce qui relèverait de la Nouvelle Objectivité, Suzanne Pagé affirme la singularité de la scène occidentale, elle en circonscrit l'originalité. L'autonomie revendiquée par rapport aux ÉtatsUnis vise quant à elle à montrer que l'Europe de l'Ouest possède les ressources pour répondre à la crise des avant-gardes et qu'elle n'a pas besoin d'un nouveau modèle universel comme celui proposé par Lothar Lang dans son texte du catalogue sur l'art de RDA. ${ }^{18}$

À travers ces expositions, on isole deux cultures allemandes distinctes, chacune marquée par des pratiques et des héritages artistiques différents. Or, cette question de la division de la culture allemande est alors au cœur des débats. Il serait présomptueux de penser que les organisateurs de l'exposition aient cherché à trancher la question, mais ce

et est étroitement lié à la politique adoptée alors par la France à l'égard de la RDA (Voir Culture et politique étrangères des démocraties populaires, Antoine Marès (dir.), Paris : Institut d'études slaves, 2007). Bernadette Contensou, texte introductif dans : Peinture et gravure en République Démocratique Allemande, cat. exp. Paris, musée d'Art moderne de la Ville de Paris, 12 mars au 26 avril 1981,n.p. : « A ce titre aussi, elle nous paraît être pour le public français une information utile et précise sur l'art, mais aussi à travers lui, sur une forme de société tendue vers un but commun et à l'intérieur de laquelle chacun apporte sa contribution dans le domaine qui est le sien.

On peut donc voir ici, par les œuvres qui nous sont proposées, une image fidèle et cohérente d'un art qui depuis trente ans participe étroitement à l'édification de la jeune République Démocratique Allemande. »

17. Hans Joachim Hoffman, avant-propos dans: Peinture et gravure en République Démocratique Allemande (note 16), n. p. : « Le réalisme, l'engagement social, la vitalité, la profondeur philosophique, la sensibilité, l'aspiration à l'efficacité et à l'action sur le plan social, la recherche du débat créateur, sont sans nul doute des motivations qui habitent de nombreux artistes. " Sans détour le texte de Lothar Lang: «De quelques particularités de la peinture et des arts graphiques en RDA », dans : Peinture et gravure en République Démocratique Allemande (note 16), n. p., définit le « réalisme socialiste » comme le point de congruence des différents langages artistiques de la RDA. Raoul-Jean Moulin : «Pour tenter d'en finir avec quelques idées reçues ", dans: Peinture et gravure en République Démocratique Allemande (note 16), n. p., établit un lien direct entre les réalismes socialistes et « la tradition réaliste de l'art allemand depuis la Renaissance, de Dürer à Dix, mais aussi par rapport à l'ébranlement expressionniste des années vingt et aux nouveaux modes de figuration qui se sont généralisés de par le monde à partir des années soixante. ”

18. Lothar Lang : «De quelques particularités de la peinture et des arts graphiques en RDA », dans : Peinture et gravure en République Démocratique Allemande (note 16), n. p. : "Les causes de l'intérêt croissant porté à l'art de la R.D.A. sont diverses et ne résident pas seulement dans la continuité remarquable d'une recherche ininterrompue, passionnée, de formes d'expressions nouvelles et réalistes, adaptée à notre temps. Il est beaucoup plus probable que l'une des causes de ce phénomène réside dans le fait que l'art de la RDA est perçu comme une contre-proposition engagée aux tendances, à la mythologie personnelle et au désengagement thématique. En d'autres termes, un large public s'intéresse de manière primaire aux idées (contenus) auxquelles l'art de la RDA donne forme dans ses œuvres. » 
qui est certain, c'est que leur choix est fortement imprégné par une certaine conception de la culture allemande contemporaine. La mise en valeur de la scène artistique allemande contemporaine dans «ses partis pris, sa radicalité et sa différence », correspond à une conception liée au bloc de l'Ouest et qui ne cherche pas à réunir, mais à déclarer un représentant officiel. C'est au même moment qu'est formulé le débat autour de la dite «question allemande » au début des années 1980, et qui soulève la question fondamentale de savoir si la division du pays était la cause essentielle du sentiment national divisé, et si seule la réunification de l'Allemagne permettrait de sortir de ce dilemme.

La très nette distinction entre l'Est et l'Ouest telle qu'elle est sensible au travers de l'exposition Art Allemagne Aujourd'hui. fait prévaloir une identité allemande occidentale. Ce discours n'est donc pas neutre dans le contexte du moment, il est même extrêmement politique et sert parfaitement la politique de Valéry Giscard d'Estaing à l'égard de l'Allemagne. La relance de la Guerre froide en 1977 avec la crise des SS20, puis la guerre d'Afghanistan en 1979, font craindre à la France que l'Allemagne, à travers son Ostpolitik, n'aille céder aux sirènes soviétiques, qui lui font miroiter une éventuelle réunification au prix d'une dérive à l'Est, d'une neutralisation et donc d'une trahison de la République fédérale envers la cause européenne occidentale. Ces suspicions ne sont cependant pas partagées par Valéry Giscard d'Estaing, qui est à la fois pour la désidéologisation des relations entre l'Est et l'Ouest, et un fervent partisan des accords d'Helsinki de 1975 qui avaient donné plus que jamais l'impression que l'Europe était figée dans sa division.

Ainsi la conception de la détente défendue par Valéry Giscard d'Estaing consiste en une consolidation du statu quo, à condition de le rendre "vivable». Les sphères d'influence doivent être bien déterminées entre les deux camps, mais on évite de se faire la guerre; on s'efforce de réduire les dangers du face à face, en développant notamment les relations commerciales. Mais il ne s'agit pas de remettre en cause les résultats de la Seconde Guerre mondiale. ${ }^{19}$

L'effort pour distinguer aussi nettement que possible chacune des scènes artistiques, la volonté de faire d'Art Allemagne Aujourd'hui le décor de la rencontre entre Valéry Giscard d'Estaing et Helmut Schmidt qui finalement échouera après que les artistes se furent élevés contre l'instrumentalisation politique de leur art en France, les mots

19. Voir notamment le numéro spécial de la revue Document, juin 1981, consacré à la question de la réunification allemande, voir également Ernst Weisenfeld : « Frankreich und die deutsche Frage ", dans : À Joseph Rovan (note 10), p. 112-122; Michèle Weinachter : Valéry Giscard d'Estaing et l'Allemagne : le double rêve inachevé, Paris, Budapest et Turin : l'Harmattan, 2004. 
de l'introduction de Bernadette Contensou au catalogue d'exposition sur la RDA qui semblent avoir été dictés par le Ministère des Affaires Étrangères attestent du caractère politique de chacune de ces manifestations culturelles et de l'effet qu'elle a exercé sur la présentation, le choix des œuvres et le discours associés à chacune des expositions.

Le tiraillement entre les identités est- et ouest-allemande permet finalement à Suzanne Pagé d'unir ce qui le plus souvent paraissait absolument diviser la génération d'avant 1968 et celle d'après 1968, pour mieux faire front contre l'Est. On comprend donc désormais plus clairement en quoi le choix des œuvres, le propos qui y est associé, sont en fait hautement déterminés par le contexte politique et donnent une orientation engagée au propos, quand bien même celui-ci n'était pas nécessairement volontaire et très largement tributaire de la langue de bois généralisée.

\section{Conclusion}

Si la presse de l'époque n'a pas saisi toute la complexité du propos de l'ARC, lequel, comme nous l'avons vu, était hautement complexe étant donné les liens étroits qu'il entretenait de manière plus ou moins ouverte et consciente avec le contexte politique et diplomatique du moment, il est cependant certain que cette exposition peut être aujourd'hui considérée comme la première présentation, si orientée soit-elle, de l'art de l'Allemagne de l'Ouest contemporaine des années 1960 et 1970 . Elle a permis au public de découvrir une scène artistique qu'il ignorait. Par la suite, les musées de Strasbourg, de Saint-Étienne, le Musée national d'art moderne - pour ne citer que quelques exemples entreprendront des acquisitions de la jeune peinture allemande. Si cela correspond bien sûr à une vogue du marché de l'époque, l'exposition Art Allemagne Aujourd'hui en a assis de toute évidence la réputation en France.

D'un point de vue culturel, cette exposition thématise de manière remarquable le sursaut identitaire qui a saisi l'Europe dans les années 1980. Après l'illusion des trente glorieuses, émerge en Europe une remise en cause fondamentale de la façon dont l'effacement des singularités a été détourné au profit de la lutte entre les blocs. 1989 fait place à une nouvelle Europe politique, et les questionnements des années 1980 sont balayés alors qu'ils contenaient des éléments très précieux pour la définition d'une identité européenne singulière qui aurait pu intégrer les singularités de la pensée des pays de l'Est, si l'on n'avait pas substitué à la logique des blocs celle, tout aussi artificielle, de la mondialisation.

Le catalogue de l'exposition Les Réalismes organisée en 1980 au Musée national d'art moderne citait une phrase tirée de la sagesse 
indienne qu'il nous semble ici pertinent de rappeler : «Qui n'est pas en mesure d'expliquer son passé est condamné à le revivre». Il est désormais fondamental d'éclairer (et de s'atteler à) la compréhension de la période de la Guerre froide pour mieux prendre conscience de tout ce que cette période nous a laissé ignorer, pour apprécier le monde dans lequel nous nous trouvons aujourd'hui, et sortir de l'idée que la logique des blocs est dépourvue de liens avec la mondialistation qui lui a fait suite. ${ }^{20}$

20. Les inquiétudes contemporaines, éclairées par Jacques Beauffet en 1980 dans le catalogue Après le classicisme, ne peuvent manquer d'évoquer celles que l'on rencontre aujourd'hui entre une mondialisation perçue comme galopante, totalisante et des résistances cherchant à défendre les particularismes, les singularités. Jacques Beauffet : « Deux ou trois choses sur la peinture, aujourd'hui », dans : Après le classicisme, cat. exp. SaintÉtienne, 1980, p. 7-8, cit. p. 8 : «Vivant encore sur la lancée «moderniste ", le public, face à ces œuvres, a parfois manifesté son incapacité à envisager autrement qu'en termes de restauration passéiste et de nationalisme de tels « retours »; partant de là, que n'a-t-on trop vite dit de la peinture de Bioulès qu'elle était «pétainiste » ou de celle de Kiefer qu'elle était « nazie ». En fait ce souci de prolonger une tradition apparaît comme la conséquence d'une volonté d'en finir avec cette espèce de fatalité de la "rupture » comme avec cette idée de «restauration », de résurrection dans l'immobilisme des modèles antérieurs qui toutes deux participent d'un même refus de l'histoire. Condamner au nom de l'internationalisme cette tentative de réinsertion des pratiques artistiques dans une continuité historique, ce retour aux racines, c'est aller un peu vite en besogne; l'internationalisme véritable ne résiderait-il pas, plutôt que dans l'artificialité terne d'une culture supra-nationale, dans la reconnaissance, l'acceptation et le respect de la différence ? À terme, on peut se demander si, dans nos sociétés où l'« action culturelle » revêt de plus en plus l'allure de la consommation de produits frelatés, l'abondance dissimulant mal l'accélération de la paupérisation des valeurs culturelles, on peut se demander si, dans un semblable contexte, ce ne sont pas de tels appels au « grand art », à la « grande culture » qui seraient aujourd'hui porteurs des valeurs réellement subversives. » (Les Réalismes 1919-1939, cat. exp., Paris, Centre Georges Pompidou, 1980, p. 6). 